\title{
Mercury Removal from Wastewater Using Palm Oil Fuel Ash
}

\author{
Imla Syafiqah Mohd Salleh ${ }^{1}$, Nor Akmalina Mustazar ${ }^{1}$, and Hafizuddin Wan Yussof ${ }^{*}$ \\ ${ }^{1}$ Faculty of Chemical Engineering \& Natural Resources, Universiti Malaysia Pahang, 26300 Gambang, Pahang, Malaysia
}

\begin{abstract}
Palm oil fuel ashes (POFA) were used as the adsorbents for the removal of $\mathrm{Hg}$ II) ions from wastewater. From the preliminary experimental results, it shows that the POFA had good adsorption capability for $\mathrm{Hg}$ (II) ions. Initially, five factors were screened using $2^{5-1}$ factorial analysis in batch mode. From these factors, only two of them gave the significant effect which are contact time and agitation speed. Both also have obtained a maximum point which is likely to be the optimum point and possible for the optimization process. This study aims to optimize these two variables (contact time and agitation speed) using central composite design (CCD) which is a subset of response surface methodology (RSM). Quadratic model was developed for $\mathrm{Hg}$ (II) percentage removals. A $2^{2}$ factorial CCD was used to get the mutual interaction between variables. The optimum adsorption conditions were obtained at contact time of $5 \mathrm{hr}$ and agitation speed of $150 \mathrm{rpm}$ with desirability of 0.95 . At these optimum points, the mercury removal efficiency was calculated at $98.93 \%$. Based on the predicted and experimental results presented, the experimental values were in good agreement with the predicted values proposed by the model with an error less than $5 \%$ and proved to be an adequate model. The results indicated that POFA has the potential to be used as an adsorbent for the removal of $\mathrm{Hg}$ (II) from aqueous environments due to its significant adsorption capacity and naturally abundance at low cost value.
\end{abstract}

\section{Introduction}

The presence of heavy metals in waste stream and ground water pose a very serious environmental concern since these metal ions are reported to be toxic to human beings as well as other living organisms. Heavy metals cause significant pollution especially for aqueous systems thus, in recent years, there has been increased research interest concerning the removal of heavy metal contaminants both in relation to improving existing methods and the development of new treatment approaches [1]. Mercury are toxic metals found in various industrial wastewaters. Several industrial activities, such as electroplating, leather tanning, metal finishing and petroleum refining, can cause water pollution by these ions. Mercury, known as a kind of remarkably toxic and nonbiodegradable metal and derives from both natural sources and industrial activities [2]. Therefore, an urgent need to effectively treat wastewater containing mercury(II) ions.

Most studies in recent years for heavy metal removal focused on the development of cheap and effective adsorbent. Researchers also looked into the availability of waste products especially on the biomass waste from agricultural to be used as an adsorbent [1] Adsorption is one the conventional technology to eliminate toxic metals from wastewater [3]. Adsorption process through utilization of adsorbents in the removal of heavy metal ions from aqueous solution is one of the most promising alternatives in substituting conventional methods like precipitation, membrane filtration, electrolyte or liquid extraction, electrodialysis and reversed osmosis [4].

Palm oil industries produce a significant amount of empty fruit bunches (EFB), oil palm fibres, and oil palm shells as wastes. POFA is a waste material obtained from burning of palm oil husk and palm kernel shell as fuel in palm oil mill boilers. The conventional method to discard these wastes is by incineration in the boilers as fuels for steam production in the oil palm mill. However, this method raises some environmental concerns due to the production of excessive amount of black soot during combustion and the production of large amounts of ash [5]. Previous studies have shown the possibility of using POFA as an adsorbent for dye removal [6], generation pf electricity [7] and as cement replacement material [8].

Adsorption method is considered flexible and easy to operate with much less sludge disposal problems. Various adsorbents such as graphene oxide [9]; coal fly ash [10]; nanoclay [11] and coconut husk [12] have been reported in the literature for the removal of mercury from wastewater. Among several chemical and physical methods, adsorption process is one of the effective techniques that have been successfully employed for mercury removal from wastewater. The main advantages of this technique are the low operating cost, improved selectivity for specific metal and short operation time. However, for industrial application, the selection of adsorbent material is mostly done by availability of

* Corresponding author: hafizuddin@ump.edu.my 
waste material and applicability of the adsorption method taking into account on space, cost, and the amount of wastewater (Mondal et al. 2013). However, new adsorbents with local availability as well as economic suitability are still needed.

The aim of this research was to study the effects of contact time and agitation speed in influencing the mercury removal efficiency using POFA as adsorbent. Respond Surface Methodology (RSM) with five replicates at the center point was performed to evaluate the contribution of the factors. The optimum conditions for the mercury removal using POFA were also determined.

\section{Materials and methods}

\subsection{Preparation of adsorbent}

POFA was collected from from palm oil mill boiler at Kilang Sawit Felda Lepar, Kuantan. Samples were washed with distilled water several times to remove impurities and then dried in an oven at $110^{\circ} \mathrm{C}$ for $24 \mathrm{~h}$. $50 \mathrm{~g}$ of POFA were activated with $0.5 \mathrm{M} \mathrm{H}_{2} \mathrm{SO}_{4}$ for 24 $\mathrm{hr}$ in a round-bottom flask. It was sieved through $100 \mu \mathrm{m}$ and stored in airtight container.

\subsection{Experimental set up}

A mercury stock solution was prepared at $1000 \mathrm{mg} / \mathrm{L}$. This stock solution was then diluted to $1 \mathrm{mg} / \mathrm{L}$ and 5 $\mathrm{mg} / \mathrm{L}$ using deionized water. All the adsorption experiment was carried out at room temperature. The required quantity of $\mathrm{Hg}^{2+}$ solution was dissolved in ultrapure water. Then, a small amount of $\mathrm{HNO}_{3}$ was added to preserve the solution. The $\mathrm{pH}$ of the solution was adjusted using $0.1 \mathrm{M}$ of $\mathrm{HCl}$ and $0.1 \mathrm{M} \mathrm{NaOH}$. The mixture of the sample is shaken by using incubator shaker at the constant room temperature. The suspension is filtered using a vacuum filter and the filtrates are analysed using the direct mercury analyser RA-3310 (Nippon Instrument Corporation, Japan). The removal efficiency of the mercury was calculated according to the following formula (equation 1):

$$
R(\%)=\left(\left(C_{o}-C_{e}\right)\right) / C_{o} \times 100
$$

where $R$ is the percent removal of mercury, $C o$ and $C e$ are the initial and residual concentration $(\mathrm{mg} / \mathrm{L})$ of mercury ion, respectively.

\subsection{Experimental design and optimization}

To understand the effect of the interaction between the adsorption-determining parameters, a design of experiment (DOE) was created with 95\% confidence limit. The optimum experimental conditions were found under response surface methodology (RSM) and performed through central composite design (CCD) using Design Expert Software, version 7.1.6 (State-Ease, Inc.). Optimization was performed using two independent variables which were identified earlier in screening process as having significant effect on mercury removal efficiency. The independent variables considered were contact time and agitation speed rpm as presented in Table 1.

The experimental data were fitted by regression to a quadratic model as mentioned in equation 2 :

$Y=\beta_{0}+\sum_{i=1}^{n} \beta_{i} X_{i}+\sum_{i=1}^{n} \beta_{i i} X_{i}^{2}+\sum_{i=1}^{n} \cdot \sum_{j=1}^{n} \beta_{i j} X_{i} X_{j}+\varepsilon$

where $Y$ represents the value of the predicted response, $\beta_{0}$ is a constant, $\beta i, \beta j$ and $\beta i j$ are the linear, quadratic and interaction coefficients, respectively, and $X i$ and $X j$ are the experimental variables which levels are being optimized.

Table 1. Levels of the mercury removal variables tested in the CCD.

\begin{tabular}{|c|c|c|c|c|c|}
\hline Factor & $-\alpha$ & -1 & 0 & +1 & $+\alpha$ \\
\hline $\begin{array}{c}\text { A: Contact } \\
\text { Time }\end{array}$ & 2 & 4.5 & 5 & 5.5 & 6 \\
\hline $\begin{array}{c}\text { B: Agitation } \\
\text { speed }\end{array}$ & 100 & 125 & 150 & 175 & 200 \\
\hline
\end{tabular}

\section{Results and discussion}

\subsection{Central composite design (CCD)}

Table 2 shows the results obtained from the 13 experiments performed through RSM approach. High mercury removal efficiency was achieved at the center point level (standard order 9-13). The second order polynomial model was utilized to express the mercury removal efficiency as a function of independent factors as shown in equation 3 :

$$
\begin{aligned}
& \text { Mercury removal efficiency }=+94.65+2.13 A \\
& -1.36 B+0.52 A B-4.52 A^{2}-6.14 B^{2}
\end{aligned}
$$

where the mercury removal efficiency represents the response, and $A$ and $B$ are the coded values of contact time and agitation speed, respectively. The term of $A$ and $B$ are denoted as the main effects, while $A B$ is the interaction involves in the mercury removal efficiency. Quadratic effects are presented through $A^{2}$ and $B^{2}$ to imply the presence of curvature in the model. This model is found to be statistically significant because its $\mathrm{p}$-value $(<0.0001)$ is less than 0.05 [13].

Table 3 presents the analysis of variance (ANOVA) and regression coefficients. A model is considered significant if the $\mathrm{p}$-values is less than 0.05 indicates that only a $5 \%$ chance of noise can occur in the model. It is apparent from the table that the model obtained was significant with $\mathrm{p}$-value is 0.0005 , while the $\mathrm{p}$-values for contact time and agitation speed are 0.0632 and 0.2010 , respectively. The p-values was lower than 0.05 , 
indicating that the model may be considered to be statistically significant [14]. Meanwhile, lack of fit is a vital expression which is express the pure error. As shown in table 3, the p-value of lack of fit (0.6332) is not significant, it means suitable and feasible model for predicting and describing the determination of $\mathrm{Hg}$ (II) ions as well as an appropriate fit. A coefficient of determination $\left(\mathrm{R}^{2}\right)$ for this study is 0.9354. It demonstrates that the predicted model perfectly satisfies the experimental data. According to [15] values for predicted and adjusted $\mathrm{R}^{2}$ strongly proof high applicability and efficiency of model.

Table 2. Experimental design and response for optimization.

\begin{tabular}{|c|c|c|c|}
\hline Std. & $\begin{array}{c}\text { Contact time } \\
\text { (hr) }\end{array}$ & $\begin{array}{c}\text { Agitation speed } \\
(\mathbf{r p m})\end{array}$ & $\begin{array}{c}\text { Mercury removal } \\
\text { efficiency } \\
(\mathbf{\%})\end{array}$ \\
\hline 1 & 4.5 & 125 & 84.67 \\
\hline 2 & 5.5 & 125 & 85.07 \\
\hline 3 & 4.5 & 175 & 79.33 \\
\hline 4 & 5.5 & 175 & 81.80 \\
\hline 5 & 4 & 150 & 71.53 \\
\hline 6 & 6 & 150 & 82.87 \\
\hline 7 & 5 & 100 & 72.67 \\
\hline 8 & 5 & 200 & 68.80 \\
\hline 9 & 5 & 150 & 93.20 \\
\hline 10 & 5 & 150 & 95.07 \\
\hline 11 & 5 & 150 & 90.20 \\
\hline 12 & 5 & 150 & 98.93 \\
\hline 13 & 5 & 150 & 98.40 \\
\hline
\end{tabular}

Table 3. Formatting sections, subsections and subsubsections.

\begin{tabular}{|c|c|c|c|c|c|c|}
\hline Source & $\mathbf{S S}^{\mathbf{A}}$ & $\mathbf{D F}^{\mathbf{B}}$ & MS $^{\mathbf{C}}$ & F-Value & $\begin{array}{c}\text { P- } \\
\text { Value }\end{array}$ & \\
\hline Model & 1133.51 & 5 & 226.70 & 20.28 & 0.0005 & Significan \\
\hline $\begin{array}{l}\text { A-Contact } \\
\text { Time }\end{array}$ & 54.40 & 1 & 54.40 & 4.87 & 0.0632 & \\
\hline $\begin{array}{l}\text { B-Agitation } \\
\text { Speed }\end{array}$ & 22.28 & 1 & 22.28 & 1.99 & 0.2010 & \\
\hline $\mathrm{Ab}$ & 1.07 & 1 & 1.07 & 0.096 & 0.7659 & \\
\hline $\mathrm{A}^{2}$ & 468.51 & 1 & 468.51 & 41.90 & 0.0003 & \\
\hline $\mathrm{B}^{2}$ & 863.29 & 1 & 863.29 & 77.21 & $\begin{array}{c}< \\
0.0001\end{array}$ & \\
\hline Residual & 78.27 & 7 & 11.18 & & & \\
\hline Lack of Fit & 25.10 & 3 & 8.37 & 0.63 & 0.6332 & $\begin{array}{c}\text { Not } \\
\text { significant }\end{array}$ \\
\hline Pure Error & 53.16 & 4 & 13.29 & & & \\
\hline Cor Total & 1211.77 & 12 & & & & \\
\hline
\end{tabular}

${ }^{\text {a}}$ Sum of squares.

${ }^{b}$ Degree of freedom.

${ }^{\mathrm{c}}$ Mean Square 


\subsection{Response surface plot}

The response surface methodology (RSM) was then applied for improving the optimization and evaluation of the relative significance and all significance interaction of variables on adsorption processes. Figure 1 and figure 2 shows the mutual interactions of the combination of self-determining variables on mercury removal efficiency in the style of 3D surface and contour plots.

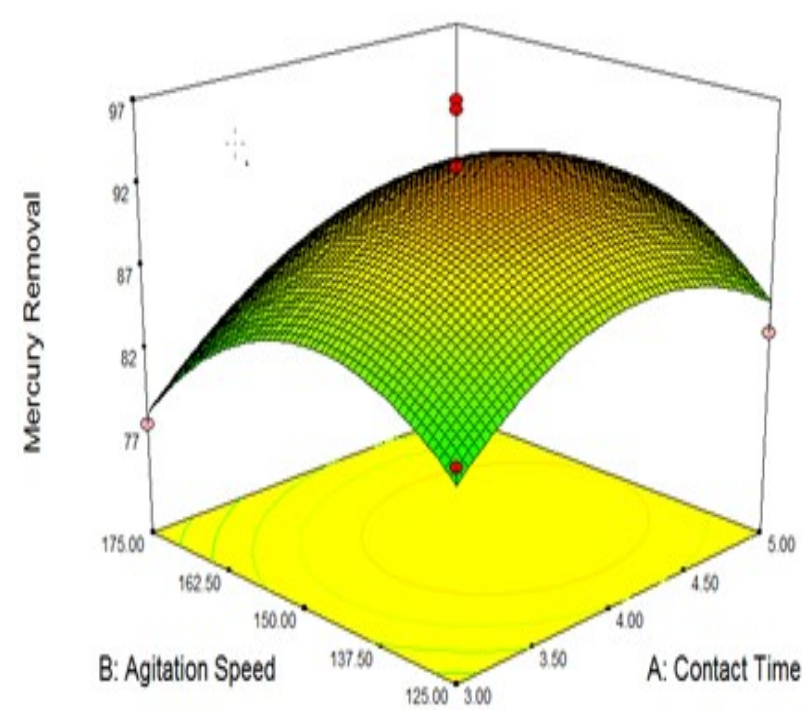

Fig. 1. 3D surface for interactive effect variables

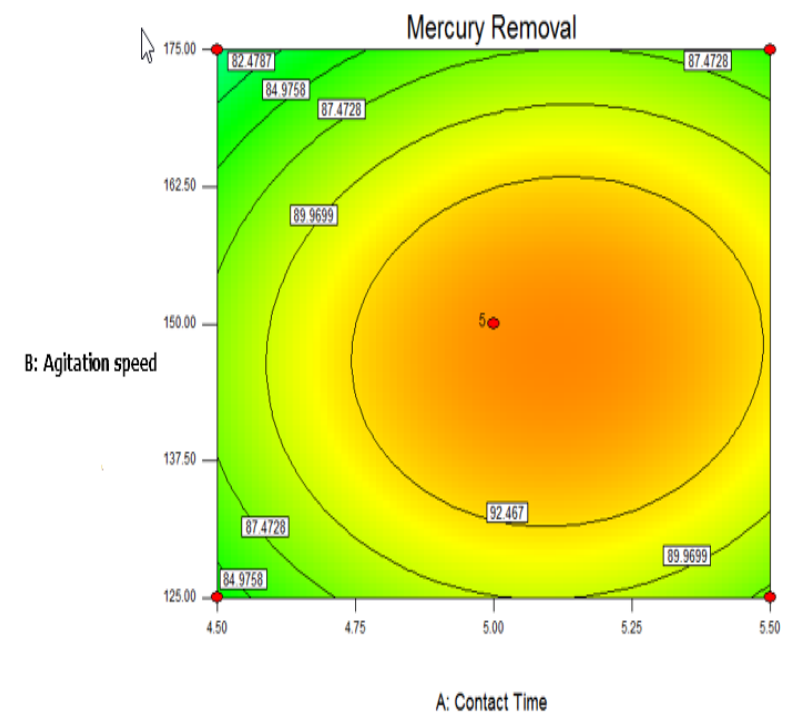

Fig. 2. Contour plots for interactive effect variables

The 3D dome-shaped curve and the 2D contour plot demonstrate the main relationship between contact time and agitation speed and the highest mercury removal efficiency. It is noticed that the percentage of mercury removal increased and reached the maximum level at contact time at $5 \mathrm{hr}$ and $150 \mathrm{rpm}$ of volume of agitation speed, and decreased beyond that. Adequacy of the model was reconfirmed by carrying out three replications at the estimated optimum point. This $3 \mathrm{D}$ plot illustrates the surface where the maximum point is placed inside the experimental region. The red zone is the optimal condition for mercury removal efficiency. At different agitation speed, the mercury removal efficiency reaches maximum value at approximate $5 \mathrm{hr}$ and slightly decreases above the value.

\section{Conclusions}

In this study, the effect of contact time and volume of agitation speed was well described by RSM. The optimum condition at $150 \mathrm{rpm}$ of agitation and $5 \mathrm{hr}$ of contact time had successfully increased the percentage of mercury removal to $98.93 \%$. A high correlation second order polynomial equation between observed and predicted responses was developed by RSM for the mercury removal efficiency. It presents the relationship between each factor and response efficiently. Therefore, it was verified that palm oil fuel ash has high potential for removing mercury in industrial wastewater.

This research was funded by Universiti Malaysia Pahang under the internal grant RDU170350. The authors would like to thank the Faculty of Chemical and Natural Resources Engineering, Universiti Malaysia Pahang for providing the infrastructure facilities for this study.

\section{References}

1. H. Xiyili, S. Çetintaş, and D. Bingöl, Process Safety and Environmental Protection, 109 288-300 (2017)

2. M. Xu, P. Yin, X. Liu, X. Dong, Y. Yang, Z. Wang and $\mathrm{R}$. Qu, Desalination and Water Treatment, 51(22-24) 4546-4555 (2013)

3. B.S. Kruthi and M.R. Rajani, International Journal of Technical Research and Applications, 3(4) 169173 (2015)

4. Z.N. Garba, I. Bello, A. Galadima, and A.Y. Lawal, Karbala, International Journal of Modern Science, 2(1) 20-28 (2016)

5. C. Acquah, L. Sie Yon, Z. Tuah, N. Ling Ngee, M.K. Danquah, Journal of Cleaner Production, 139 10981104 (2016)

6. W.A. Khanday, F. Marrakchi, M. Asif, B.H. Hameed, Journal of the Taiwan Institute of Chemical Engineers, 70 32-41(2017).

7. B.S. Thomas, S. Kumar, H.S. Arel, Renewable and Sustainable Energy Reviews, 80 550-561 (2017).

8. S. Pourakbar, A. Asadi, B.B.K. Huat, M.H. Fasihnikoutalab, Transportation Geotechnics, 3 24-35 (2015)

9. T. Esfandiyari, N. Nasirizadeh, M. Dehghani and M.H. Ehrampoosh, Chinese Journal of Chemical Engineering, 25(9) 1170-1175 (2017)

\footnotetext{
* Corresponding author: hafizuddin@ump.edu.my
} 
10. M. Attari, S.S. Bukhari, H. Kazemian, and S. Rohani, Journal of Environmental Chemical Engineering, 5(1) 391-399 (2017)

11. M. Soleimani, and Z.H. Siahpoosh, Chinese Journal of Chemical Engineering, 23(11) 1819-1833 (2015).

12. K. Johari, N. Saman, S.T. Song, C.S. Chin, H. Kong, and H. Mat, International Biodeterioration \& Biodegradation, 109 45-52 (2016).

13. D.O. Aksoy and E. Sagol, Fuel, 183 609-616 (2016)

14. J. Aravind, C. Lenin, C. Nancyflavia, P. Rashika and S. Saravanan, International Journal of Environmental Science and Technology, 12(1), 105-114 (2015)

15. K. Dashtian and R. Zare-Dorabei, Journal of Colloid and Interface Science, 494 114-123 (2017) 